\title{
Expression of CLDN6 in tissues of gastric cancer patients: Association with clinical pathology and prognosis
}

\author{
FANGMEI GAO ${ }^{1}$, MINGDONG LI $^{2}$, RUI XIANG ${ }^{2}$, XIN ZHOU $^{2}$, LIANYING ZHU $^{2}$ and YI ZHAI ${ }^{3}$ \\ Departments of ${ }^{1}$ Gastroenterology (I), ${ }^{2}$ Gastroenterology (II) and ${ }^{3}$ Oncology (I), \\ Central Hospital of Zibo, Zibo, Shandong 255036, P.R. China
}

Received September 21, 2018; Accepted February 22, 2019

DOI: $10.3892 /$ ol.2019.10129

\begin{abstract}
Expression of claudin-6 (CLDN6) in the tissues of gastric cancer patients and its association with clinical pathology and prognosis were investigated. A retrospective analysis was performed on 213 gastric cancer patients diagnosed and surgically treated in the Central Hospital of Zibo from January 2010 to January 2013. Cancer and normal adjacent tissues were obtained from the patients to detect the expression level of CLDN6 using reverse transcription-quantitative PCR (RT-qPCR). The association between the expression level of CLDN6 and the clinical and pathological features, as well as the prognosis of gastric cancer patients was analyzed. The expression level of CLDN6 was significantly lower in gastric cancer tissues than that in adjacent tissues $(\mathrm{t}=23.350, \mathrm{P}<0.001)$. The expression level of CLDN6 was associated with age, lymph node metastasis, pathological staging, and distant metastasis $(\mathrm{P}<0.05)$. In this study, patients were separated into CLDN6 high-expression group $(\geq 1.42)$ with 107 patients and CLDN6 low-expression group $(<1.42)$ with 106 patients, with the median expression level of CLDN6 as the boundary. The 1-, 2- and 3-year survival rates of patients in the CLDN6 low-expression group were 80.19, 60.38 and $48.11 \%$, respectively, and those in the CLDN6 high-expression group were $87.85,73.83$ and $66.36 \%$, respectively. The survival rate was significantly better in the CLDN6 high-expression group than that in the CLDN6 low-expression group $(\mathrm{P}=0.009)$. In conclusion, the expression level of CLDN6 is low in the cancer tissues of gastric cancer patients, and associated with age, lymph node metastasis, pathological staging and distant metastasis. CLDN6 low expression has a certain negative impact on the prognosis of patients, and therefore, shows potential as an important indicator for the prognosis of gastric cancer patients.
\end{abstract}

Correspondence to: Dr Yi Zhai, Department of Oncology (I), Central Hospital of Zibo, 54 Gongqingtuan West Road, Zibo, Shandong 255036, P.R. China

E-mail: zecq3n@163.com

Key words: CLDN6, gastric cancer, expression, clinical pathology, prognosis

\section{Introduction}

Gastric cancer, one of the most common digestive tract malignancies in the world, accounts for $10-15 \%$ of systemic malignancies (1). According to the statistics reported by Correa (2), there were $\sim 4$ million new gastric cancer patients around the world in 2015, with an increasing incidence observed year by year. Gastric cancer is more common in developed countries in Europe and North America, among which the United States have the highest incidence (3). Based on the study by Lee et al (4), the incidence of gastric cancer will exceed $50 \%$ in the next 50 years, and gastric cancer will become the malignant tumor with the highest incidence. Gastric cancer is more common in people $>50$ years of age, and the males to females incidence ratio is 2:1 (5). As diet structure and living habits change, the onset age of gastric cancer has become younger, which has seriously affected the social stability and labor (6). There is no obvious symptom in the early stage of gastric cancer, so it is often ignored by patients, who thereby miss the best treatment period, which leads to high prognostic mortality (7). According to the study by Shimada et al (8), the prognostic 5-year survival rate of gastric cancer patients is only $55.4 \%$. Because of its high incidence and mortality, gastric cancer remains a hot research topic in clinic.

Claudins (CLDNs), the abnormal expression of which causes structural changes in epithelial or endothelial cells and damages cell function, play an important role especially in cardiovascular, metabolic and digestive system diseases (9). At present, there is a relevant study (10) reporting that CLDNs, including claudin-6 (CLDN6), are involved in the occurrence and development of multiple tumors. In the study by Zhang et al, CLDN6 has been proven to be closely related to cervical cancer (11). CLDN6 is also specifically expressed in gastric cancer. In this study, an experimental analysis was carried out to investigate whether CLDN6 can be a diagnostic or therapeutic indicator for gastric cancer, in order to provide an effective and reliable reference for the treatment of gastric cancer patients.

\section{Patients and methods}

Clinical information of patients. A retrospective analysis was performed on 213 gastric cancer patients diagnosed and 
surgically treated in the Central Hospital of Zibo (Zibo, China) from January 2010 to January 2013. Patients included 98 females and 115 males, with an average age of $48.2 \pm 3.6$ years. There were 75 patients in stage I-II and 138 patients in stage III-IV. During the operation, gastric cancer and adjacent tissues $(5 \mathrm{~cm}$ away from the cancerous tissues) were taken and stored at $-80^{\circ} \mathrm{C}$. Inclusion criteria: cancer tissue specimens diagnosed by pathology, and adjacent tissues diagnosed as no cancer or inflammatory cell infiltration; patients with complete medical records and follow-up data. Exclusion criteria: patients who had undergone radiotherapy and chemotherapy before the specimens were taken, pregnant or lactating patients, patients with other severe diseases and patients with communication or cognitive disorders. This study was approved by the Ethics Committee of Central Hospital of Zibo. Patients who participated in this research had complete clinical data and cooperated with the medical staff to complete the relevant medical treatment. Signed informed consents were obtained from all patients or their guardians. The basic information of patients is shown in Table I.

Experimental apparatus and materials. Quantitative PCR instrument was purchased from Bio-Rad Laboratories, Inc. (Hercules, CA, USA), TRIzol reagent from Shanghai Mingjin Biotech Co., Ltd. (Shanghai, China) (cat. no. 53011000), RT-qPCR kit from Shanghai GenePharma Co., Ltd. (Shanghai, China) (cat. no. QPG-020-QPG-023), reverse transcription kit from Shanghai Hifun Biotechnology Co., Ltd. (Shanghai, China) (cat. no. A0005), and NanoDrop 2000 UV spectrophotometer from Thermo Fisher Scientific, Inc. (Waltham, MA, USA).

RT-qPCR detection of CLDN6 expression. TRIzol reagent was added to the gastric cancer and adjacent tissues for extracting RNA in order to detect its purity and concentration using the UV spectrophotometer. Total RNA $(1 \mu \mathrm{g})$ was taken to reverse transcribe cDNA in strict accordance with the kit instructions. Reaction parameters were as follows: $16^{\circ} \mathrm{C}$ for $15 \mathrm{~min}, 42^{\circ} \mathrm{C}$ for $60 \mathrm{~min}$, and $85^{\circ} \mathrm{C}$ for $5 \mathrm{~min}$. The transcribed cDNA was used for PCR amplification. The PCR amplification system was configured according to the manufacturer's instructions of SYBR ${ }^{\mathrm{TM}}$ Green Master Mix (Thermo Fisher Scientific, Inc.), with U6 as an internal reference. Primer sequences are shown in Table II. PCR reaction conditions were as follows: pre-denaturation at $95^{\circ} \mathrm{C}$ for $5 \mathrm{~min}$, and then at $95^{\circ} \mathrm{C}$ for $20 \mathrm{sec}$ and at $65^{\circ} \mathrm{C}$ for $45 \mathrm{sec}$ for a total of 45 cycles. The RT-qPCR instrument was used for detection. CLDN6 primer sequence was designed and synthesized by Sangon Biotech Co., Ltd. (Shanghai, China), with U6 as a reaction internal reference. Each group of samples was repeatedly measured 3 times, and $2^{-\mathrm{ACq}}$ was used to analyze the expression level of CLDN6 in the specimens (12).

Statistical analysis. SPSS 18.6 statistical software (Beijing Strong-Vinda Information Technology Co., Ltd., Beijing, China) was used for analyzing and processing the data. The basic enumeration data of patients were expressed as percentage [n (\%)]. The expression level of CLDN6 was expressed as mean \pm standard deviation (mean $\pm \mathrm{SD}$ ), and was statistically analyzed by t-test. ANOVA was used for comparison between multiple groups with LSD post hoc test.
Table I. General information of 213 gastric cancer patients [n (\%)].

\begin{tabular}{lr}
\hline Factor & $\mathrm{n}=213$ \\
\hline Sex & \\
Male & $115(53.99)$ \\
Female & $98(46.01)$ \\
Age (years) & \\
$\leq 50$ & $86(40.38)$ \\
$>50$ & $127(59.62)$ \\
Smoking & \\
Yes & $168(78.87)$ \\
No & $45(21.13)$ \\
Drinking & \\
Yes & $146(68.54)$ \\
No & $67(31.46)$ \\
Pathological staging & \\
Stage I-II & $75(35.21)$ \\
Stage III-IV & $138(64.79)$ \\
Pathological classification & \\
Adenocarcinoma & $76(35.68)$ \\
Adenosquamous carcinoma & $61(28.64)$ \\
Squamous carcinoma & $39(18.31)$ \\
Carcinoid & $37(17.37)$ \\
Classification of disease sites & \\
Gastric fundus and cardia cancer & $95(44.60)$ \\
Gastric corpus cancer & $65(30.52)$ \\
Gastric antrum cancer & $53(24.88)$ \\
\hline
\end{tabular}

Kaplan-Meier was used for calculating the survival rate, and log-rank test was used for comparison of the survival curves. $\mathrm{P}<0.05$ was considered to indicate a statistically significant difference.

\section{Results}

Comparison of CLDN6 expression between gastric cancer and adjacent tissues. The results of RT-qPCR detection of CLDN6 showed that the expression level of CLDN6 was $1.34 \pm 0.26$ in the gastric cancer tissues and $2.58 \pm 0.73$ in the adjacent tissues. The expression of CLDN6 was significantly lower in the gastric cancer tissues than that in the adjacent tissues, with a statistically significant difference $(\mathrm{t}=23.350$, $\mathrm{P}<0.001)$ (Fig. 1).

Association of CLDN6 expression level with clinical and pathological features of gastric cancer patients. There was no significant difference in the expression level of CLDN6 between male and female patients. The expression level of CLDN6 was not associated with the sex of gastric cancer patients, with no statistically significant difference $(\mathrm{P}>0.05)$. The expression of CLDN6 was lower in patients $>50$ years of age than that in patients $\leq 50$ years of age, with a statistically significant difference $(\mathrm{P}<0.05)$. Expression of CLDN6 
Table II. Primer sequences of CLDN6 and internal reference.

\begin{tabular}{lll}
\hline Genes & Upstream primer & Downstream primer \\
\hline CLDN6 & 5'-TTCATCGGCAACAGCATCGT-3' & 5'-GGTTATAGAAGTCCCGGATGA-3' \\
U6 & 5'-GCTTCGGCAGCACATATACTAAAAT-3' & 5'-CGCTTCACGAATTTGCGTGTCAT-3'
\end{tabular}

CLDN6, claudin-6.

Table III. Association of CLDN6 expression level with clinical and pathological features of gastric cancer patients.

\begin{tabular}{|c|c|c|c|c|}
\hline Clinical and pathological features & $\mathrm{n}=213$ & CLDN6 (ng/ml) & t (statistical value) & P-value (statistical difference) \\
\hline Sex & & & & 0.663 \\
\hline Male & 115 & $1.35 \pm 0.18$ & 0.436 & \\
\hline Female & 98 & $1.34 \pm 0.15$ & & \\
\hline Age (years) & & & & $<0.001$ \\
\hline$\leq 50$ & 86 & $1.42 \pm 0.17$ & 4.672 & \\
\hline$>50$ & 127 & $1.28 \pm 0.24$ & & \\
\hline Lymph node metastasis & & & & $<0.001$ \\
\hline Yes & 154 & $1.27 \pm 0.21$ & 4.629 & \\
\hline No & 59 & $1.41 \pm 0.16$ & & \\
\hline Pathological staging & & & & $<0.001$ \\
\hline Stage I-II & 75 & $1.39 \pm 0.11$ & 4.939 & \\
\hline Stage III-IV & 138 & $1.24 \pm 0.25$ & & \\
\hline Distant metastasis & & & & $<0.001$ \\
\hline Yes & 132 & $1.25 \pm 0.22$ & 5.420 & \\
\hline No & 81 & $1.41 \pm 0.19$ & & \\
\hline Location classification of the disease & & & & 0.461 \\
\hline Gastric fundus and cardia cancer & 95 & $1.31 \pm 0.22$ & 0.777 & \\
\hline Gastric corpus cancer & 65 & $1.23 \pm 0.31$ & & \\
\hline Gastric antrum cancer & 53 & $1.26 \pm 0.18$ & & \\
\hline
\end{tabular}

CLDN6, claudin-6.

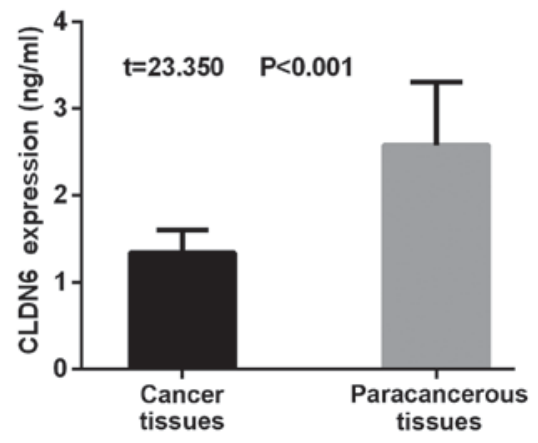

Figure 1. Comparison of CLDN6 expression between gastric cancer and adjacent tissues. The results of RT-qPCR showed that the expression level of CLDN6 was significantly lower in the gastric cancer tissues than that in the adjacent tissues, with a statistically significant difference $(\mathrm{t}=23.350$, $\mathrm{P}<0.001)$. CLDN6, claudin-6.

was lower in patients with lymph node metastasis than that in patients without lymph node metastasis, with a statistically significant difference $(\mathrm{P}<0.05)$. CLDN6 expression was significantly lower in patients with stage III-IV than that in patients with stage I-II, with a statistically significant difference $(\mathrm{P}<0.05)$. CLDN6 expression was also significantly lower in patients with distant metastasis than that in patients without distant metastasis, with a statistically significant difference $(\mathrm{P}<0.05)$. There was no statistically significant difference in the expression level of CLDN6 among patients with gastric corpus cancer, gastric antrum cancer and gastric fundus and cardia cancer $(\mathrm{P}>0.05)$ (Table III).

Association of CLDN6 expression level with prognosis of gastric cancer patients. Patients were divided into CLDN6 high-expression group $(\geq 1.42)$ with 107 patients and CLDN6 low-expression group $(<1.42)$ with 106 patients, with the median expression level of CLDN6 as the boundary line. A 3-year follow-up survey was performed on the patients by telephone, review and letter, until June 2018 or the death of the patient. The 1-, 2- and 3-year survival rates of patients in the 


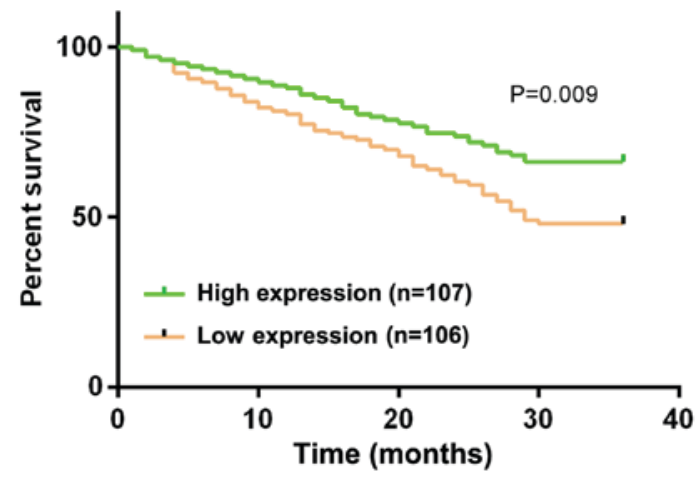

Figure 2. Association of CLDN6 expression level with prognosis of gastric cancer patients. Kaplan-Meier was used to calculate the survival rate and log-rank test was used for the comparison of the survival curves. In this experiment, patients were divided into the CLDN6 high-expression group $(\geq 1.42)$ with 107 patients and the CLDN6 low-expression group $(<1.42)$ with 106 patients, with the median expression level of CLDN6 as the boundary. The 1-, 2- and 3-year survival rates of patients in the CLDN6 low-expression group were $80.19,60.38$ and $48.11 \%$, respectively, and those in the CLDN6 high expression group were $87.85,73.83$ and $66.36 \%$, respectively. CLDN6, claudin-6.

CLDN6 low-expression group were 80.19, 60.38 and 48.11\%, respectively, and those in the CLDN6 high-expression group were $87.85,73.83$ and $66.36 \%$, respectively. The survival rate of patients was significantly higher in the CLDN6 high-expression group than that in the CLDN6 low-expression group ( $\mathrm{P}=0.009)$ (Fig. 2).

\section{Discussion}

Gastric cancer is currently a major malignant tumor worldwide, and its high incidence and mortality are among the top ranking in malignancies (13). With changes in lifestyle and diet structure and the increase in social pressure, the onset age of gastric cancer is gradually becoming younger (14). Gastric cancer originates from the gastric mucosa epithelium, and there is no symptom with obvious specificity in the early stage of most of gastric cancer patients. Abdominal distension and abdominal pain, which are easily confused with symptoms of gastritis, gastric ulcer and other chronic stomach diseases, are often ignored by patients $(15,16)$. At present, the diagnostic rate of early gastric cancer is low, which seriously affects the prognosis of gastric cancer patients (17). Therefore, finding a factor with high sensitivity and good accuracy for gastric cancer has become a research hotspot in clinical practice.

CLDN6 is a family member of CLDNs which contains 4 transmembrane domains (18). It protects epithelial cells and is important for the occurrence and development of tumors (9). In this study, a retrospective analysis was performed on 213 gastric cancer patients diagnosed and surgically treated from January 2010 to January 2013. The cancer and adjacent tissues were taken from the patients in order to investigate the association of CLDN6 expression in the tissues with the prognosis of patients. First, CLDN6 expression was compared between the gastric cancer and the adjacent tissues in gastric cancer patients, and the association of CLDN6 expression level with the clinical and pathological features of the patients was analyzed. The results of RT-qPCR detection showed that the expression level of CLDN6, which was significantly lower in the gastric cancer tissues than that in the adjacent tissues, was associated with age, lymph node metastasis, pathological staging and distant metastasis, with statistically significant differences. There is a recent study showing that CLDN6 expression is low in breast cancer tissues (19). In the study by Guo et al (20), CLDN6 expression is low in breast cancer cells and closely related to the proliferation and invasion of the cells, which corroborates our findings. The association of CLDN6 expression level with the prognosis of gastric cancer patients was also investigated in the present study, and a 3-year follow-up survey was performed on patients by telephone, review and letter. The 1-,2- and 3-year survival rates of patients in the CLDN6 low-expression group were $80.19,60.38$ and $48.11 \%$, respectively, and those in the CLDN6 high-expression group were 87.85, 73.83 and 66.36\%, respectively. The survival rate of patients was significantly higher in the CLDN6 high-expression group than that in the CLDN6 low-expression group. The prognosis of gastric cancer is related to its pathological staging, location, tissue type, biological behavior and treatment measures (21). According to Torres-Martínez et al (22) there is a correlation of CLDN6 with gastric cancer, the expression is associated with the development of tumors, affecting the nutrition of cells and the supply of szomatomedin, thereby increasing the proliferation ability of tumor cells. In this study, it was found that the lower the expression of CLDN6, the worse the prognosis of gastric cancer patients was. It is indicated that CLDN6 can be used as an indicator for the prognosis of gastric cancer patients. Monitoring the expression level of CLDN6 can improve the prognosis of the disease.

There are still some limitations in this study. There may be differences in the expression of CLDN6 among gastric cancer patients of different ages. Also, a longer-time follow-up survey of the subjects should also be conducted.

In conclusion, the expression level of CLDN6 is low in cancer tissues of gastric cancer patients, and associates with age, lymph node metastasis, pathological staging and distant metastasis. CLDN6 low expression has a certain negative impact on the prognosis of patients, and shows potential as an important indicator for the prognosis of gastric cancer patients.

\section{Acknowledgements}

Not applicable.

\section{Funding}

No funding was received.

\section{Availability of data and materials}

The datasets used and/or analyzed during the current study are available from the corresponding author on reasonable request.

\section{Authors' contributions}

FG and ML performed PCR. FG and YZ were involved in the writing of the manuscript. RX and XZ acquired and analyzed 
the general data of patients. LZ and YZ assisted with pathology and prognosis analysis. All authors read and approved the final manuscript.

\section{Ethics approval and consent to participate}

This study was approved by the Ethics Committee of Central Hospital of Zibo (Zibo, China). Patients who participated in this research had complete clinical data. Signed informed consents were obtained from the patients or their guardians.

\section{Patient consent for publication}

Not applicable.

\section{Competing interests}

The authors declare that they have no competing interests.

\section{References}

1. Van Cutsem E, Sagaert X, Topal B, Haustermans K and Prenen H: Gastric cancer. Lancet 388: 2654-2664, 2016.

2. Correa P: Diet and gastric cancer. In: Diet, Nutrition and Cancer: A Critical Evaluation. Reddy BS and Cohen AL (eds). Vol 1. 1st edition. CRC Press, pp1-10, 2017.

3. Oki E, Tokunaga S, Emi Y, Kusumoto T, Yamamoto M, Fukuzawa K, Takahashi I, Ishigami S, Tsuji A, Higashi H, et al; Kyushu Study Group of Clinical Cancer: Surgical treatment of liver metastasis of gastric cancer: A retrospective multicenter cohort study (KSCC1302). Gastric Cancer 19: 968-976, 2016.

4. Lee YC, Chiang TH, Chou CK, Tu YK, Liao WC, Wu MS and Graham DY: Association between Helicobacter pylori eradication and gastric cancer incidence: A systematic review and meta-analysis. Gastroenterology 150: 1113-1124.e5, 2016.

5. Karimi P, Islami F, Anandasabapathy S, Freedman ND and Kamangar F: Gastric cancer: Descriptive epidemiology, risk factors, screening, and prevention. Cancer Epidemiol Biomarkers Prev 23: 700-713, 2014.

6. Kim JW, Nam KH, Ahn SH, Park DJ, Kim HH, Kim SH, Chang H, Lee JO, Kim YJ, Lee HS, et al: Prognostic implications of immunosuppressive protein expression in tumors as well as immune cell infiltration within the tumor microenvironment in gastric cancer. Gastric Cancer 19: 42-52, 2016.

7. Yamada T, Yoshikawa T, Taguri M, Hayashi T, Aoyama T, Sue-Ling HM, Bonam K, Hayden JD and Grabsch HI: The survival difference between gastric cancer patients from the UK and Japan remains after weighted propensity score analysis considering all background factors. Gastric Cancer 19: 479-489, 2016.
8. Shimada H, Noie T, Ohashi M, Oba K and Takahashi Y: Clinical significance of serum tumor markers for gastric cancer: A systematic review of literature by the Task Force of the Japanese Gastric Cancer Association. Gastric Cancer 17: 26-33, 2014.

9. Sahin U, Tureci O, Koslowski M, Walter K, Woll S, Kreuzberg M, Hubner B and Erdeljan M: Antibodies specific for claudin 6 (CLDN6): US Patent 9,487,584. Filed November 11, 2010; issued August 11, 2016.

10. Nelhúbel GA, Károly B, Szabó B, Lotz G, Kiss A, Tóvári J and Kenessey I: The prognostic role of claudins in head and neck squamous cell carcinomas. Pathol Oncol Res 20: 99-106, 2014.

11. Zhang WN, Li W, Wang XL, Hu Z, Zhu D, Ding WC, Liu D, Li KZ, Ma D and Wang H: CLDN1 expression in cervical cancer cells is related to tumor invasion and metastasis. Oncotarget 7: 87449-87461, 2016.

12. Livak KJ and Schmittgen TD: Analysis of relative gene expression data using real time quantitative PCR and the 2(-Delta Delta C(T)) method. Methods 25: 402-408, 2001.

13. Ferro A, Peleteiro B, Malvezzi M, Bosetti C, Bertuccio P, Levi F, Negri E, La Vecchia $C$ and Lunet N: Worldwide trends in gastric cancer mortality (1980-2011), with predictions to 2015, and incidence by subtype. Eur J Cancer 50: 1330-1344, 2014.

14. Rugge M, Fassan M and Graham DY: Epidemiology of gastric cancer. In: Gastric Cancer. Strong VE (ed.). 1st edition. Springer International Publishing, Switzerland, pp23-34, 2015.

15. Sano T: Gastric cancer: Asia and the world. Gastric Cancer 20 (Suppl 1): 1-2, 2017.

16. Graham DY: Helicobacter pylori update: Gastric cancer, reliable therapy, and possible benefits. Gastroenterology 148: 719-31.e3, 2015.

17. Merchant SJ, Kim J, Choi AH, Sun V, Chao J and Nelson R: A rising trend in the incidence of advanced gastric cancer in young Hispanic men. Gastric Cancer 20: 226-234, 2017.

18. Arabzadeh A, Troy TC and Turksen K: Role of the Cldn6 cytoplasmic tail domain in membrane targeting and epidermal differentiation in vivo. Mol Cell Biol 26: 5876-5887, 2006.

19. Yang M, Li Y, Shen X, Ruan Y, Lu Y, Jin X, Song P, Guo Y, Zhang X, Qu H, et al: CLDN6 promotes chemoresistance through GSTP1 in human breast cancer. J Exp Clin Cancer Res 36: 157, 2017.

20. Guo Y, Lin D, Zhang M, Zhang X, Li Y, Yang R, Lu Y, Jin X, Yang M, Wang M, et al: CLDN6-induced apoptosis via regulating ASK1-p38/JNK signaling in breast cancer MCF-7 cells. Int J Oncol 48: 2435-2444, 2016

21. Maruyama K: The most important prognostic factors for gastric cancer patients: A study using univariate and multivariate analyses. Scand J Gastroenterol 22 (Suppl 133): 63-68, 1987.

22. Torres-Martínez AC, Gallardo-Vera JF, Lara-Holguin AN, Montaño LF and Rendón-Huerta EP: Claudin-6 enhances cell invasiveness through claudin-1 in AGS human adenocarcinoma gastric cancer cells. Exp Cell Res 350: 226-235, 2017.

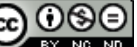

This work is licensed under a Creative Commons Attribution-NonCommercial-NoDerivatives 4.0 International (CC BY-NC-ND 4.0) License. 